\title{
OPTOFLUIDICS
}

\section{Tunable microlenses}

Optofluidic microlenses of tunable focal length would aid applications in photolithography, optical imaging and on-chip cell sorting and detection. Current techniques for tuning microlenses, through the use of an electric field or electrowetting, for example, often cause optical aberrations, liquid evaporation, slow response times and narrow tuning ranges.

Now, by exploiting multilayer soft lithography - a technique widely used to fabricate highly integrated large-scale microfluidic chips Yanyi Huang and co-workers from Peking University in China have developed a compound microlens that is flexible, robust and tunable (Lab Chip 11, 2835-2841; 2011).

The researchers formed a chip containing many microchannels from three horizontal layers of the organic polymer polydimethylsiloxane. Each layer contained a round chamber connected to one of the channels, through which liquid could be injected. In the middle layer, several rectangular chambers were connected to the 700- $\mu$ m-diameter round chamber to function as integrated pneumatic valves.

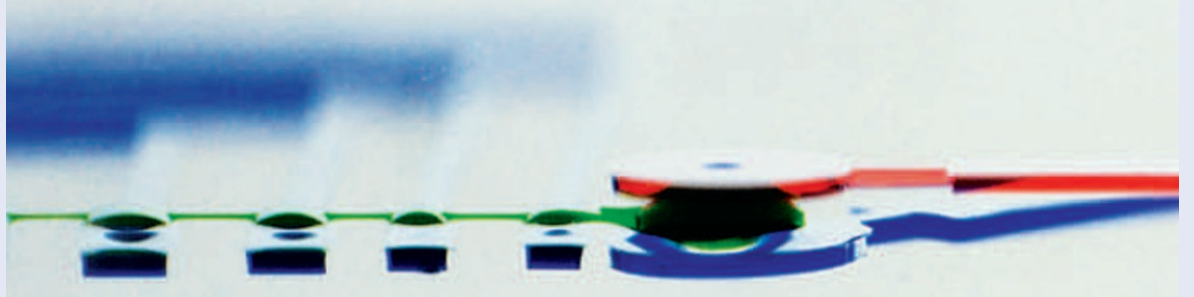

Actuation of the valves was used to determine the volume of liquid present in the chambers and thus to tune the focal length of the microlens. The researchers say that the refractive index of the microlens can be adjusted by filling the chambers with different liquids.

The compound microlens has several attractive properties, including selfalignment, large zoom ratio $(\sim 7 x)$, a widely tunable angle of view $\left(15-80^{\circ}\right)$, large focal range (from submillimetres to to 0.44), small lens size (a few hundred micrometres in diameter) and a zooming actuation response time of around $100 \mathrm{~ms}$.

According to Huang, the fabrication method not only meets many of the challenges faced by optofluidic microlens builders for over a decade, but also opens many new possibilities for constructing other types of tunable micro-optical components. centimetres), large numerical aperture (up

RACHEL WON

\section{QUANTUM COMMUNICATION}

\section{Super-activated channels}

In recent years, significant progress has been made towards putting information theory on the footing of quantum mechanics. Researchers have now shown how optical fibres with zero quantum capacity can be super-activated to allow for perfect quantum communication.

\section{Geza Giedke and Michael M. Wolf}

E ver since the birth of quantum mechanics at the beginning of the twentieth century, researchers have been astounded by the range of paradoxical or 'spooky' phenomena found throughout the quantum world. Some of the most important quantum paradoxes were pointed out by the fathers of the field; Einstein, Podolsky and Rosen were puzzled with what seemed to be 'spooky action at a distance', and Schrödinger stumbled over a cat with a mysterious quantum life-line.
From a distance, these phenomena are often considered to be a modern manifestation of Aristotle's principle that "the whole is greater than the sum of its parts." Now, writing in Nature Photonics, Smith, Smolin and Yard ${ }^{1}$ demonstrate a particularly striking example of such an effect in the context of quantum communication: two channels that are themselves useless for quantum communication can allow perfect transmission if used together. This demonstration is remarkable both in terms of its simplicity and its applications to communication technology. The researchers use Gaussian channels, which, needless to say, form the foundation of classical communication, for instance in the form of lossy optical fibres.

The effect demonstrated by Smith et al. can be regarded as a typical example of a broader class of phenomena occurring in the field of quantum information science - namely the non-additivity of basic resources and standard units. Classically, basic resources in physics, 